Journal of Mathematics and Informatics

Vol. 19, 2020, 47-56

ISSN: 2349-0632 (P), 2349-0640 (online)

Published 9 September 2020

www.researchmathsci.org

DOI: http://dx.doi.org/10.22457/jmi.v19a06180

Journal of

Mathematics and

Informatics

\title{
Cubic Rational B-Spline Curves with Two Parameters
}

Ji Peipei, Zhang Guicang and Wang Kai

School of Mathematics and Statistics, Northwest Normal University,

Lanzhou 730070, China

*Corresponding author. Email: $1286594820 @$ qq.com

Received 14 July 2020; accepted 2 September 2020

\begin{abstract}
In view of the problems existing in the traditional literature to improve the traditional B-spline method, such as: the parameters are not global, the method is not general and can not retain the important properties of the traditional B-spline method. A non-uniform B-spline basis with two parameters is proposed to construct curve. Firstly, we assume that the non-uniform cubic rational B-spline basis possessing $C^{2}$ continuity and partition of unity at each knot, and then the expression of the basis function can be determined; then the important properties of the basis function like totally positive are proved; The definition of non-uniform cubic rational B-spline curve is given and its important properties such as variation diminishing are proved. The example shows that the non-uniform B-spline curve constructed in this paper effectively solves the problems existing in the traditional improved method and is suitable for curve design.
\end{abstract}

Keywords: B-spline; non-uniform; basis function; totally positive; variation diminishing

AMS Mathematics Subject Classification (2010): 41A15

\section{Introduction}

The classical B-spline method is proposed under the condition of preserving the advantages of Bézier method and overcoming the disadvantage of non local property caused by global representation and solving the connection problem in describing complex shapes. But the problem of its weight factor has not been solved. For this purpose, many scholars have proposed quasi B-spline curves with shape parameters [1-14].

At present, there are many improvements on the classical B-spline method, and the uniform B-spline method is mainly used, but this kind of method has not been widely used in geometric modeling design. Next, because the results of cubic B-spline curves are simple and not ineffective, they are discussed most in the literature. In reference [15], 
Ji Peipei, Zhang Guicang and Wang Kai

Chen Fulai et al. Gave a class of cubic polynomial basis functions with two parameters. On the basis of this basis function, this paper constructs a class of non-uniform cubic rational B-spline basis, and gives the definition of non-uniform cubic rational B-spline curve, and proves its important properties such as variation reduction and local control. Experiments show that the non-uniform B-spline curve constructed in this paper solves the problem of flexibility of traditional B-spline curve. In addition, when the control points are determined, the curve can still be modified locally.

\section{The definition and properties of base}

\subsection{Preliminary knowledge}

The basis function $\left(u_{0}, u_{1}, \ldots, u_{n}\right)$ is called the total positive basis on the closed interval $[a, b]$. If for any node sequence $a \leq t_{0}<t_{1}<\cdots<t_{n} \leq b$, the collocation matrix $\left(u_{i}\left(t_{i}\right)\right)_{0 \leq i, j \leq n}$ of the basis function is a totally positive matrix, that is, the determinant of any submatrix of the allocation matrix is nonnegative. For a function space with totally positive bases, a set of totally positive bases can be multiplied by the total positive transformation matrix to generate the remaining totally positive bases [16].

\subsection{Construction of nonuniform cubic triangular B-spline bases}

A class of cubic rational polynomial basis functions with two parameters are constructed in reference [15].

$$
\left\{\begin{array}{l}
A_{0}(t)=(1-t)^{2}(1-\alpha t) \\
A_{1}(t)=(2+\alpha) t(1-t)^{2} \\
A_{2}(t)=(2+\beta) t^{2}(1-t) \\
A_{3}(t)=(1-\beta+\beta t) t^{2}
\end{array}\right.
$$

Among them, $\alpha, \beta \in(-2,1], t \in[0,1]$. Since the basis function $\left\{A_{i}(t), i=0,1,2,3\right\}$ is a set of positive bases, the corresponding B é zier curves generated by B é zier functions have important properties such as variation reduction, which is suitable for curve design. In view of the fact that the quasi B é zier curves defined by (1) do not have local control, and the problem of smooth splicing needs to be solved when designing complex curves; therefore, on the basis of (1), this paper constructs a class of non-uniform Cubic Rational B-spline bases (CR-B-splines) for curve design.

For a given node sequence $u_{0}<u_{1}<\cdots<u_{n+4}$, we denote it as node vector $U=\left(u_{0}, u_{1}, \cdots, u_{n+4}\right)$, let $h_{j}=u_{j+1}-u_{j}, t_{j}(u)=\left(u-u_{j}\right) / h_{j}, j=0,1, \cdots, n+3$, for any shape parameter $\alpha_{i}, \beta_{i} \in(-1 / 2,1], i=0,1, \cdots, n$, the CR-B spline base to be constructed is 
Cubic Rational B-Spline Curves with Two Parameters

$$
B_{i}(u)= \begin{cases}B_{i, 0}\left(t_{i}\right)=d_{i} A_{3}\left(t_{i} ; \beta_{i}\right), & u \in\left[u_{i}, u_{i+1}\right), \\ B_{i, 1}\left(t_{i+1}\right)=\sum_{j=0}^{3} c_{i+1, j} A_{j}\left(t_{i+1}, \alpha_{i+1}, \beta_{i+1}\right), & u \in\left[u_{i+1}, u_{i+2}\right), \\ B_{i, 2}\left(t_{i+2}\right)=\sum_{j=0}^{3} b_{i+2, j} A_{j}\left(t_{i+2}, \alpha_{i+2}, \beta_{i+2}\right), & u \in\left[u_{i+2}, u_{i+3}\right),(2) \\ B_{i, 3}\left(t_{i+3}\right)=a_{i+3} A_{0}\left(t_{i+3} ; \alpha_{i+3}\right), & u \in\left[u_{i+3}, u_{i+4}\right), \\ 0, & u \notin\left[u_{i}, u_{i+4}\right) .\end{cases}
$$

where $A_{j}\left(t_{i} ; \alpha_{i}, \beta_{i}\right), j=0,1,2,3$ is the basis function given in formula (1).

In order to determine the value of coefficient $a_{i}, b_{i, j}, c_{i, j}, d_{i}$, the CR-B spline basis constructed is restricted by two conditions: (1) $B_{i}(u)$ is $C^{2}$-Continuous on all nodes; (2) $B_{i}(u)$ is unit on interval $\left[u_{3}, u_{n+1}\right]$. After some calculation, the coefficient can be directly obtained as follows:

$$
\begin{aligned}
& \lambda_{i}=\left(2 \alpha_{i+1}+1\right)\left(\beta_{i}+2\right) h_{i}+\left(\alpha_{i+1}+2\right)\left(2 \beta_{i}+1\right) h_{i+1} ; \mu_{i}=\left(\alpha_{i+1}+2\right) h_{i}+\left(\beta_{i}+2\right) h_{i+1} ; \\
& \phi_{i}=\frac{\lambda_{i} h_{i}+3 \mu_{i} h_{i+1}}{3\left(\beta_{i}+2\right) h_{i+1}^{2}} ; \varphi_{i}=\frac{\lambda_{i-1} h_{i}+3 \mu_{i-1} h_{i-1}}{3\left(\alpha_{i}+2\right) h_{i-1}^{2}} ; \\
& a_{i}=\frac{3\left(\beta_{i-1}+2\right) \lambda_{i-2} h_{i}^{2}}{3 \lambda_{i-1} \mu_{i-2} h_{i-2}+\lambda_{i-2} \lambda_{i-1} h_{i-1}+3 \lambda_{i-2} \mu_{i-1} h_{i}} ; d_{i}=\frac{3\left(\alpha_{i+1}+2\right) \lambda_{i+1} h_{i}^{2}}{3 \lambda_{i+1} \mu_{i} h_{i}+\lambda_{i} \lambda_{i+1} h_{i+1}+3 \lambda_{i} \mu_{i+1} h_{i+2}} ; \\
& b_{i, 0}=\frac{\left(\alpha_{i}+2\right) \phi_{i} h_{i-1}}{\mu_{i-1}} a_{i+1}+\frac{\left(\beta_{i-1}+2\right) \varphi_{i-1} h_{i}}{\mu_{i-1}} d_{i-2} ; b_{i, 1}=\phi_{i} a_{i+1} ; c_{i, 0}=d_{i-1} ; \\
& b_{i, 2}=\frac{\mu_{i}}{\left(\beta_{i}+2\right) h_{i+1}} a_{i+1} ; c_{i, 1}=\frac{\mu_{i-1}}{\left(\alpha_{i}+2\right) h_{i-1}} d_{i-1} ; b_{i, 3}=a_{i+1} ; c_{i, 2}=\varphi_{i} d_{i-1} ; \\
& c_{i, 3}=\frac{\left(\alpha_{i+1}+2\right) \phi_{i+1} h_{i}}{\mu_{i}} a_{i+2}+\frac{\left(\beta_{i}+2\right) \varphi_{i} h_{i+1}}{\mu_{i}} d_{i-1} .
\end{aligned}
$$

Definition 1. Given the node vector $U$, for any $\alpha_{i}, \beta_{i} \in(-1 / 2,1]$ and the coefficient $a_{i}, b_{i, j}, c_{i, j}, d_{i}$ given above, (2) is called CR-B spline basis with two parameters.

For equidistant nodes, CR-B spline base $B_{i}(u)$ is called uniform CR-B spline base, and corresponding node $U$ vector is called isometric node vector. For non-uniform nodes, CR-B spline basis $B_{i}(u)$ is called non-uniform CR-B spline basis, and corresponding node vector $U$ is called non-uniform node vector. Figure 1 shows the images of uniform CR-B spline basis under different shape parameters. 
Ji Peipei, Zhang Guicang and Wang Kai
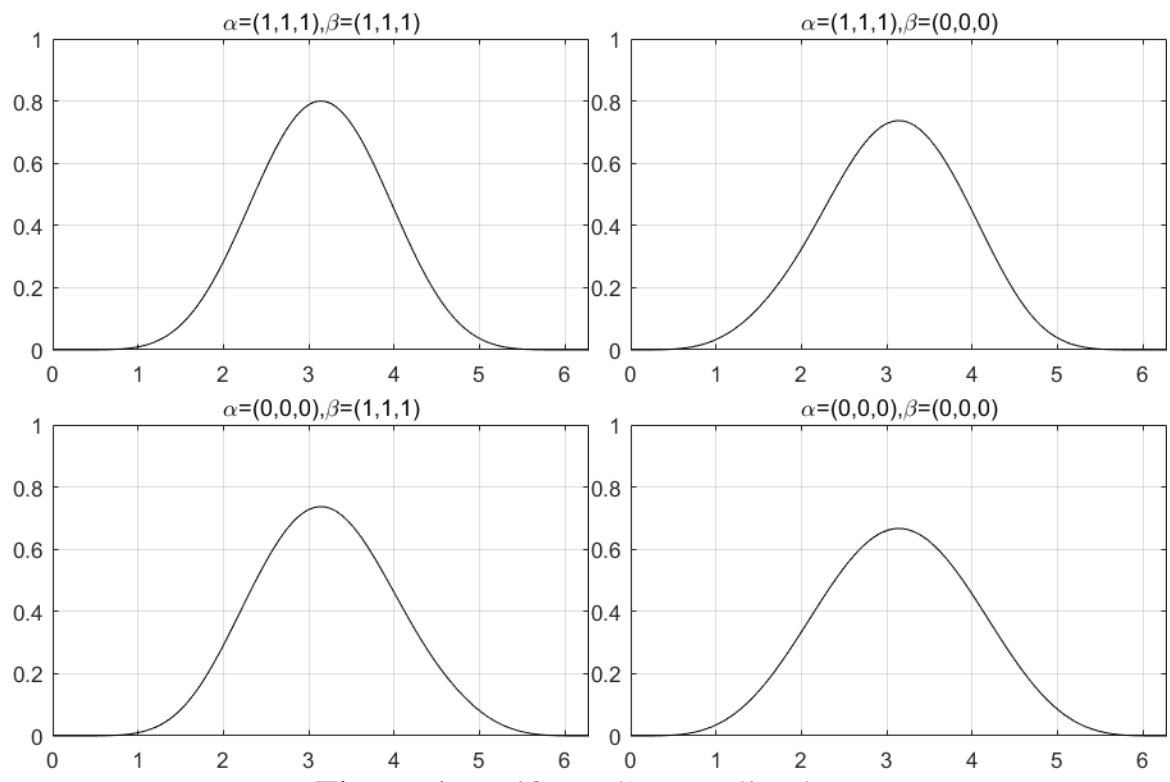

Figure 1: Uniform CR-B spline base

The following lemma can be obtained by direct calculation, which will be very useful for the following discussion.

Lemma 1. For any $i \in Z$, the following equation holds

(1) $a_{i}+b_{i 0}+c_{i 0}=1 ;(2) b_{i 1}+c_{i 1}=1 ;(3) b_{i 2}+c_{i 2}=1 ;(4) b_{i 3}+c_{i 3}+d_{i}=1$;

(5) $d_{i}=c_{i+1,0} ;(6) b_{i+2,0}=c_{i+1,3} ;(7) b_{i+2,3}=a_{i+3}$;

(8) $\left(\frac{1}{h_{i}}\right)\left(\beta_{i}+2\right) d_{i}=\left(\frac{1}{h_{i+1}}\right)\left(\alpha_{i+1}+2\right)\left(c_{i+1,1}-c_{i+1,0}\right)$;

(9) $\left(\frac{1}{h_{i+1}}\right)\left(\beta_{i+1}+2\right)\left(c_{i+1,3}-c_{i+1,2}\right)=\left(\frac{1}{h_{i+2}}\right)\left(\alpha_{i+2}+2\right)\left(b_{i+2,1}-b_{i+2,0}\right)$;

(10) $\left(\frac{1}{h_{i+2}}\right)\left(\beta_{i+2}+2\right)\left(b_{i+2,3}-b_{i+2,2}\right)=-\left(\frac{1}{h_{i+3}}\right)\left(\alpha_{i+3}+2\right) a_{i+3}$;

(11) $\left(\frac{1}{h_{i}}\right)^{2}\left(4 \beta_{i}+2\right) d_{i}=\left(\frac{1}{h_{i+1}}\right)^{2}\left[\left(4 \alpha_{i+1}+2\right) c_{i+1,0}-\left(4 \alpha_{i+1}+8\right) c_{i+1,1}+\left(4+2 \beta_{i}\right) c_{i+1,2}+\left(2-2 \beta_{i}\right) c_{i+1,3}\right]$;

(12) $\left(\frac{1}{h_{i+1}}\right)^{2}\left[\left(2-2 \alpha_{i+1}\right) c_{i+1,0}+\left(4+2 \alpha_{i+1}\right) c_{i+1,1}-\left(4 \beta_{i}+8\right) c_{i+1,2}+\left(4 \beta_{i}+2\right) c_{i+1,3}\right]$

$=\left(\frac{1}{h_{i+2}}\right)^{2}\left[\left(4 \alpha_{i+2}+2\right) b_{i+2,0}-\left(4 \alpha_{i+2}+8\right) b_{i+2,1}+\left(4+2 \beta_{i+2}\right) b_{i+2,2}+\left(2-2 \beta_{i+2}\right) b_{i+2,3}\right]$;

(13) $\left(\frac{1}{h_{i+2}}\right)^{2}\left[\left(2-\alpha_{i+2}\right) b_{i+2,0}+\left(4+2 \alpha_{i+2}\right) b_{i+2,1}-\left(4 \beta_{i+2}+8\right) b_{i+2,2}+\left(4 \beta_{i+2}+2\right) b_{i+2,3}\right]=\left(\frac{1}{h_{i+3}}\right)^{2}\left(4 \alpha_{i+3}+2\right) a_{i+3}$. 


\section{Cubic Rational B-Spline Curves with Two Parameters}

Note 1: The CR-B spline basis function is constructed in the function space $T=\left\{s \in C^{2}\left[u_{0}, u_{n+4}\right], s_{i}=\left.s\right|_{\left[u_{i}, u_{i+1}\right]} \in T_{\alpha_{i}, \beta_{i}}, i=0,1, \ldots, n+3\right\}$, where $T_{\alpha_{i}, \beta_{i}}=\operatorname{span}\left\{1,3 t^{2}-2 t^{3},(1-t)^{2}(1-\alpha t)\right.$,

$\left.(1-\beta+\beta t) t^{2}\right\}, \quad t_{j}(u)=\left(u-u_{j}\right) / h_{j}, j=0,1, \cdots, n+3$. when the uniform node vector $U$ and $\alpha_{i+1}=\alpha_{i+2}=\alpha_{i+3}=\beta_{i}=\beta_{i+1}=\beta_{i+2}=1$ are given, the CR-B spline basis is the classical non-uniform cubic B-spline basis [17]. In particular, for $u_{i+j}=u_{i}+j h, h>0, j=1,2,3,4$, direct calculation can be used

$$
\begin{array}{llllll}
d_{i}=\frac{1}{6} & c_{i+1,0}=\frac{1}{6} & c_{i+1,1}=\frac{1}{3} & c_{i+1,2}=\frac{2}{3} & c_{i+1,3}=\frac{2}{3} \\
b_{i+1,0}=\frac{2}{3} & b_{i+1,1}=\frac{2}{3} & b_{i+1,2}=\frac{1}{3} & b_{i+1,3}=\frac{1}{6} & a_{i+3}=\frac{1}{6}
\end{array}
$$

The CR-B-spline basis is a classical uniform cubic B-spline basis.

\subsection{Properties of nonuniform CR-B spline basis}

Theorem 1. Normative: for arbitrary $u \in\left[u_{3}, u_{n+1}\right], \quad \sum_{i=0}^{n} B_{i}(u)=1$.

Proof: For $u \in\left[u_{i}, u_{i+1}\right), i=3,4, \cdots, n$, obviously $B_{j}(u)=0, j \neq i-3, i-2, i-1, i$. Due to

$$
\begin{array}{ll}
B_{i-3}(u)=a_{i} A_{0}\left(t_{i} ; \alpha_{i}\right), & B_{i-2}(u)=\sum_{j=0}^{3} b_{i, j} A_{j}\left(t_{i} ; \alpha_{i}, \beta_{i}\right), \\
B_{i-1}(u)=\sum_{j=0}^{3} c_{i, j} A_{j}\left(t_{i} ; \alpha_{i}, \beta_{i}\right), & B_{i}(u)=d_{i} A_{3}\left(t_{i}, \beta_{i}\right),
\end{array}
$$

Therefore, using lemma 1 , we can get

$$
\sum_{i=0}^{n} B_{i}(u)=a_{i} A_{0}\left(t_{i} ; \alpha_{i}\right)+\sum_{j=0}^{3} b_{i, j} A_{j}\left(t_{i} ; \alpha_{i}, \beta_{i}\right)+\sum_{j=0}^{3} c_{i, j} A_{j}\left(t_{i} ; \alpha_{i}, \beta_{i}\right)+d_{i} A_{3}\left(t_{i} ; \beta_{i}\right)=\sum_{j=0}^{3} A_{j}\left(t_{i} ; \beta_{i}\right)=1 .
$$

Conclusion.

Theorem 2. Nonnegativity: if $\alpha_{i}, \beta_{i} \in(-1 / 2,1]$, then for any $u_{i}<u<u_{i+4}$, there is $B_{i}(u)>0$.

Proof: It is proved that for $\alpha_{i}, \beta_{i} \in(-1 / 2,1]$, obviously $a_{i}, b_{i, j}, c_{i, j}, d_{i}>0, B_{i}(u)>0$ can be obtained by the nonnegativity of $A_{j}\left(t_{i} ; \alpha_{i}, \beta_{i}\right), j=0,1,2,3^{[16]}$.

Theorem 3. Linear independence: linear independence on interval $\left[u_{3}, u_{n+1}\right]$ for any $\alpha_{i}, \beta_{i} \in(-1 / 2,1],\left\{B_{0}(u), B_{1}(u), \cdots, B_{n}(u)\right\}$.

Proof: Take $\xi_{i} \in R(i=0,1, \cdots, n), u \in\left[u_{3}, u_{n+1}\right]$, consider $B(u)=\sum_{i=0}^{n} \xi_{i} B_{i}(u)=0$ for any $\alpha_{i}, \beta_{i} \in(-1 / 2,1]$, have 
Ji Peipei, Zhang Guicang and Wang Kai

$$
\begin{aligned}
& B\left(u_{i}\right)=a_{i} \xi_{i-3}+b_{i, 0} \xi_{i-2}+c_{i, 0} \xi_{i-1}=0, \\
& B^{\prime}\left(u_{i}\right)=\frac{1}{h_{i}}\left[\left(\alpha_{i}+2\right) a_{i}\left(\xi_{i-2}-\xi_{i-3}\right)+\frac{\left(\beta_{i-1}+2\right) d_{i-1} h_{i}}{h_{i-1}}\left(\xi_{i-1}-\xi_{i-2}\right)\right]=0 \\
& B^{\prime \prime}\left(u_{i}\right)=\left(\frac{1}{h_{i}}\right)^{2}\left[2\left(2 \alpha_{i}+1\right) a_{i}\left(\xi_{i-3}-\xi_{i-2}\right)+\frac{2\left(2 \beta_{i-1}+1\right) d_{i-1} h_{i}^{2}}{h_{i-1}^{2}}\left(\xi_{i-1}-\xi_{i-2}\right)\right]=0
\end{aligned}
$$

where $i=3,4, \cdots, n+1$, the following linear equations can be obtained

$$
\left\{\begin{array}{l}
a_{i}\left(\xi_{i-3}-\xi_{i-2}\right)+\left(a_{i}+b_{i, 0}+c_{i, 0}\right) \xi_{i-2}+c_{i, 0}\left(\xi_{i-1}-\xi_{i-2}\right)=0, \\
-\left(\alpha_{i}+2\right) a_{i}\left(\xi_{i-3}-\xi_{i-2}\right)+\frac{\left(\beta_{i-1}+2\right) d_{i-1} h_{i}}{h_{i-1}}\left(\xi_{i-1}-\xi_{i-2}\right)=0, \\
\left(2 \alpha_{i}+1\right) a_{i}\left(\xi_{i-3}-\xi_{i-2}\right)+\frac{\left(2 \beta_{i-1}+1\right) d_{i-1} h_{i}^{2}}{h_{i-1}^{2}}\left(\xi_{i-1}-\xi_{i-2}\right)=0 .
\end{array}\right.
$$

From $a_{i}+b_{i 0}+c_{i 0}=1$, then the corresponding coefficient determinant is

$$
\begin{gathered}
\left|M_{i}\right|=\left|\begin{array}{ccc}
a_{i} & 1 & c_{i, 0} \\
-\left(\alpha_{i}+2\right) a_{i} & 0 & \frac{\left(\beta_{i-1}+2\right) d_{i-1} h_{i}}{h_{i-1}} \\
\left(2 \alpha_{i}+1\right) a_{i} & 0 & \frac{\left(2 \beta_{i-1}+1\right) d_{i-1} h_{i}^{2}}{h_{i-1}^{2}}
\end{array}\right| \\
=\frac{a_{i} d_{i-1} h_{i}}{h_{i-1}^{2}}\left[\left(2 \beta_{i-1}+1\right) h_{i}\left(\alpha_{i}+2\right)+\left(2 \alpha_{i}+1\right)\left(\beta_{i-1}+2\right) h_{i-1}\right]>0 . \\
\text { So } \xi_{i-3}=\xi_{i-2}=\xi_{i-1}=0, i=3,4, \ldots, n+1 . \text { The proof is over. }
\end{gathered}
$$

Theorem 4. Totally positive: for $u \in\left[u_{i}, u_{i+1}\right], \alpha_{i}, \beta_{i} \in(-1 / 2,1], i=3,4, \ldots, n$, $\left(B_{i-3}(u), B_{i-2}(u), B_{i-1}(u), B_{i}(u)\right)$ constitutes a set of normal totally positive bases in space $T_{\alpha_{i}, \beta_{i}}$.

Proof: For $u \in\left[u_{i}, u_{i+1}\right], i=3,4, \ldots, n$, that is

$$
\begin{aligned}
& \left(B_{i-3}(u), B_{i-2}(u), B_{i-1}(u), B_{i}(u)\right)=\left(A_{0}\left(t_{i} ; \alpha_{i}\right), A_{1}\left(t_{i} ; \alpha_{i}\right), A_{2}\left(t_{i} ; \beta_{i}\right), A_{3}\left(t_{i} ; \beta_{i}\right)\right) H_{i} \text {, where } \\
& H_{i}=\left[\begin{array}{cccc}
a_{i} & b_{i, 0} & c_{i, 0} & 0 \\
0 & b_{i, 1} & c_{i, 1} & 0 \\
0 & b_{i, 2} & c_{i, 2} & 0 \\
0 & b_{i, 3} & c_{i, 3} & d_{i}
\end{array}\right], \alpha_{i}, \beta_{i} \in(-1 / 2,1], t_{i}(u)=\left(u-u_{i}\right) / h_{i},
\end{aligned}
$$

Since $\left(A_{0}\left(t_{i} ; \alpha_{i}\right), A_{1}\left(t_{i} ; \alpha_{i}\right), A_{2}\left(t_{i} ; \beta_{i}\right), A_{3}\left(t_{i} ; \beta_{i}\right)\right)$ is a total positive basis in function space $T_{\alpha_{i}, \beta_{i}}$, it is only necessary to prove that $\left(B_{i-3}(u), B_{i-2}(u), B_{i-1}(u), B_{i}(u)\right)$ constitutes a set of normal totally positive bases in function space $T_{\alpha_{i}, \beta_{i}}$ by proving that the transformation matrix $H_{i}$ is a nonsingular random totally positive matrix. For any $\alpha_{i}, \beta_{i} \in(-1 / 2,1]$, there is obviously $a_{i}, b_{i, j}, c_{i, j}, d_{i}>0$. From lemma 1 , we can see that the 
Cubic Rational B-Spline Curves with Two Parameters

sum of each row of $H_{i}$ is 1 , that is, $H_{i}$ is a random matrix. In addition, direct calculation includes

$$
\begin{aligned}
\left|\begin{array}{ll}
b_{i, 0} & c_{i, 0} \\
b_{i, 1} & c_{i, 1}
\end{array}\right|= & \frac{\left(\beta_{i-1}+2\right) \varphi_{i-1} h_{i}}{\left(\alpha_{i}+2\right) h_{i-1}} d_{i-2} d_{i-1}>0, \\
\left|\begin{array}{ll}
b_{i, 0} & c_{i, 0} \\
b_{i, 2} & c_{i, 2}
\end{array}\right|= & \frac{h_{i}\left(3 \lambda_{i-1} \mu_{i} h_{i+1}+\lambda_{i-1} \lambda_{i} h_{i}+3 \lambda_{i} \mu_{i-1} h_{i-1}\right)}{9\left(\beta_{i}+2\right) \mu_{i-1} h_{i-1} h_{i+1}^{2}} a_{i+1} d_{i-1}+\frac{\left(\beta_{i-1}+2\right) \varphi_{i-1} \varphi_{i} h_{i}}{\mu_{i-1}} d_{i-2} d_{i-1}>0 \\
\left|\begin{array}{ll}
b_{i, 0} & c_{i, 0} \\
b_{i, 3} & c_{i, 3}
\end{array}\right|= & \frac{\left(\alpha_{i}+2\right)\left(\alpha_{i+1}+2\right) \phi_{i} \phi_{i+1} h_{i-1} h_{i}}{\mu_{i-1} \mu_{i}} a_{i+1} a_{i+2}+\frac{h_{i}\left(3 \lambda_{i-1} \mu_{i} h_{i+1}+\lambda_{i-1} \lambda_{i} h_{i}+3 \lambda_{i} \mu_{i-1} h_{i-1}\right)}{9 \mu_{i-1} \mu_{i} h_{i-1} h_{i+1}} a_{i+1} d_{i-1} \\
& +\frac{\left(\alpha_{i+1}+2\right)\left(\beta_{i-1}+2\right) \phi_{i+1} \varphi_{i-1} h_{i}^{2}}{\mu_{i-1} \mu_{i}} a_{i+2} d_{i-2}+\frac{\left(\beta_{i-1}+2\right)\left(\beta_{i}+2\right) \varphi_{i-1} \varphi_{i} h_{i} h_{i+1} d_{i-2} d_{i-1}>0,}{\mu_{i-1} \mu_{i}} \\
\left|\begin{array}{ll}
b_{i, 1} & c_{i, 1} \\
b_{i, 2} & c_{i, 2}
\end{array}\right|= & \frac{h_{i}\left(3 \lambda_{i-1} \mu_{i} h_{i+1}+\lambda_{i-1} \lambda_{i} h_{i}+3 \lambda_{i} \mu_{i-1} h_{i-1}\right)}{9\left(\alpha_{i}+2\right)\left(\beta_{i}+2\right) h_{i-1}^{2} h_{i+1}^{2}} a_{i+1} d_{i-1}>0, \\
\left|\begin{array}{ll}
b_{i, 1} & c_{i, 1} \\
b_{i, 3} & c_{i, 3}
\end{array}\right|= & \frac{h_{i}\left(3 \lambda_{i-1} \mu_{i} h_{i+1}+\lambda_{i-1} \lambda_{i} h_{i}+3 \lambda_{i} \mu_{i-1} h_{i-1}\right)}{9\left(\alpha_{i}+2\right) \mu_{i} h_{i-1}^{2} h_{i+1}} a_{i+1} d_{i-1}+\frac{\left(\alpha_{i+1}+2\right) \phi_{i} \phi_{i+1} h_{i}}{\mu_{i}} a_{i+1} a_{i+2}>0, \\
\left|\begin{array}{ll}
b_{i, 2} & c_{i, 2} \\
b_{i, 3} & c_{i, 3}
\end{array}\right|= & \frac{\left(\alpha_{i+1}+2\right) \phi_{i+1} h_{i}}{\left(\beta_{i}+2\right) h_{i+1}} a_{i+1} a_{i+2}>0 .
\end{aligned}
$$

It is easy to prove that $H_{i}$ is a nonsingular totally positive matrix. Therefore, the conclusion can be proved.

Theorem 5. Continuity: given a non-uniform node vector, when the shape parameter satisfies $\alpha_{i}, \beta_{i} \in(-1 / 2,1]$, the basis function $B_{i}(u)$ has continuity at each node $C^{2}$. Proof: Consider the continuity at node $u_{i+1}$, so for any $\alpha_{i}, \beta_{i} \in(-1 / 2,1]$, we have

$$
\begin{aligned}
& B_{i}\left(u_{i+1}^{-}\right)=d_{i} ; B_{i}\left(u_{i+1}^{+}\right)=c_{i+1,0} ; B_{i}^{\prime}\left(u_{i+1}^{-}\right)=\left(\frac{1}{h_{i}}\right)\left(\beta_{i}+2\right) d_{i} ; \\
& B_{i}^{\prime}\left(u_{i+1}^{+}\right)=\left(\frac{1}{h_{i+1}}\right)\left(\alpha_{i+1}+2\right)\left(c_{i+1,1}-c_{i+1,0}\right) ; B_{i}^{\prime \prime}\left(u_{i+1}^{-}\right)=\left(\frac{1}{h_{i}}\right)^{2}\left(4 \beta_{i}+2\right) d_{i} ; \\
& B_{i}^{\prime \prime}\left(u_{i+1}^{+}\right)=\left(\frac{1}{h_{i+1}}\right)^{2}\left[\left(4 \alpha_{i+1}+2\right) c_{i+1,0}-\left(4 \alpha_{i+1}+8\right) c_{i+1,1}+\left(4+2 \beta_{i+1}\right) c_{i+1,2}+\left(2-2 \beta_{i+1}\right) c_{i+1,3}\right] .
\end{aligned}
$$

From the above expression and lemma 1, we have $B_{i}\left(u_{i+1}^{+}\right)=B_{i}\left(u_{i+1}^{-}\right)$, $B_{i}^{\prime}\left(u_{i+1}^{+}\right)=B_{i}^{\prime}\left(u_{i+1}^{-}\right), B_{i}^{\prime \prime}\left(u_{i+1}^{+}\right)=B_{i}^{\prime \prime}\left(u_{i+1}^{-}\right)$theorem to prove.

\section{Non uniform cubic triangular B-spline curve}

\subsection{Definition and properties of nonuniform cubic trigonometric B-spline curves}

Definition 2. Given a non-uniform node vector $U$ and control point $P_{i}(i=0,1, \ldots, n) \in R^{2} / R^{3}, n \geq 3$, for any $\alpha_{i}, \beta_{i} \in(-1 / 2,1], n \geq 3, u \in\left[u_{3}, u_{n+1}\right]$, we say

$$
Q(u)=\sum_{i=0}^{n} B_{i}(u) P_{i}(3)
$$

is called non-uniform cubic rational B-spline curve with two parameters $\alpha_{i}, \beta_{i}$ 
Ji Peipei, Zhang Guicang and Wang Kai

(non-uniform CR-B-spline curve).

Obviously, for $u \in\left[u_{i}, u_{i+1}\right], i=3,4, \ldots, n$, , the non-uniform CT-B spline segment $B_{i}(u)$ can be expressed as follows

$$
\begin{aligned}
Q_{i}(u) & =\sum_{j=i-3}^{i} B_{j}(u) P_{j}=\left(a_{i} P_{i-3}+b_{i 0} P_{i-2}+c_{i 0} P_{i-1}\right) A_{0}\left(t_{i} ; \alpha_{i}\right)+\left(b_{i 1} P_{i-2}+c_{i 1} P_{i-1}\right) A_{1}\left(t_{i} ; \alpha_{i}\right) \\
& +\left(b_{i 2} P_{i-2}+c_{i 2} P_{i-1}\right) A_{2}\left(t_{i} ; \beta_{i}\right)+\left(b_{i 3} P_{i-2}+c_{i 3} P_{i-1}+d_{i} P_{i}\right) A_{3}\left(t_{i} ; \beta_{i}\right)
\end{aligned}
$$

According to theorems 1 and 2, for $u \in\left[u_{i}, u_{i+1}\right], i=3,4, \ldots, n$, the non-uniform CR-B spline curve $B(u)$ is affine invariant, and $B(u)$ is in the convex hull formed by the control point $P_{i-3}, P_{i-2}, P_{i-1}, P_{i}$. In addition, it can be seen from theorem 4 that the non-uniform CR-B spline curve $Q(u)$ has important variation reduction property. Therefore, the non-uniform CR-B-spline curve retains all the basic properties of the traditional B-spline, and has flexible shape adjustability, which is suitable for geometric design. The following theorem can be obtained from Theorem 5 .

Theorem 6. Given a non-uniform node vector, when the shape parameter satisfies $\alpha_{i}, \beta_{i} \in(-1 / 2,1]$, the non-uniform CR-B spline curve has $C^{2}$-continuity at each node.

Proof: In this paper, we first consider the continuity of non-uniform CR-B-spline curve at node $u_{i+1}$. According to equation (4), on the interval $\left[u_{i}, u_{i+1}\right]$ and $\left[u_{i+1}, u_{i+2}\right]$, the non-uniform CR-B spline curve segments can be expressed as

and

$$
\begin{aligned}
Q_{i}(u) & =\sum_{j=i-3}^{i} B_{j}(u) P_{j}=\left(a_{i} P_{i-3}+b_{i 0} P_{i-2}+c_{i 0} P_{i-1}\right) A_{0}\left(t_{i} ; \alpha_{i}\right)+\left(b_{i 1} P_{i-2}+c_{i 1} P_{i-1}\right) A_{1}\left(t_{i} ; \alpha_{i}\right) \\
& +\left(b_{i 2} P_{i-2}+c_{i 2} P_{i-1}\right) A_{2}\left(t_{i} ; \beta_{i}\right)+\left(b_{i 3} P_{i-2}+c_{i 3} P_{i-1}+d_{i} P_{i}\right) A_{3}\left(t_{i} ; \beta_{i}\right), \quad u \in\left[u_{i}, u_{i+1}\right]
\end{aligned}
$$

$$
\begin{aligned}
Q_{i+1}(u) & =\sum_{j=i-2}^{i+1} B_{j}(u) P_{j}=\left(a_{i+1} P_{i-2}+b_{i+1,0} P_{i-1}+c_{i+1,0} P_{i}\right) A_{0}\left(t_{i+1} ; \alpha_{i+1}\right)+\left(b_{i+1,1} P_{i-1}+c_{i+1,1} P_{i}\right) A_{1}\left(t_{i+1} ; \alpha_{i+1}\right) \\
& +\left(b_{i+1,2} P_{i-1}+c_{i+1,2} P_{i}\right) A_{2}\left(t_{i+1} ; \beta_{i+1}\right)+\left(b_{i+1,3} P_{i-1}+c_{i+1,3} P_{i}+d_{i+1} P_{i+1}\right) A_{3}\left(t_{i+1} ; \beta_{i+1}\right), u \in\left[u_{i+1}, u_{i+2}\right]
\end{aligned}
$$

So

$$
\begin{aligned}
& Q_{i}\left(u_{i+1}^{+}\right)=b_{i 3} P_{i-2}+c_{i 3} P_{i-1}+d_{i} P_{i} ; Q_{i+1}\left(u_{i+1}^{-}\right)=a_{i+1} P_{i-2}+b_{i+1,0} P_{i-1}+c_{i+1,0} P_{i} ; \\
& Q_{i}^{\prime}\left(u_{i+1}^{+}\right)=-\left(b_{i 2} P_{i-2}+c_{i 2} P_{i-1}\right)\left(\beta_{i}+2\right)+\left(b_{i 3} P_{i-2}+c_{i 3} P_{i-1}+d_{i} P_{i}\right)\left(\beta_{i}+2\right) ; \\
& Q_{i+1}^{\prime}\left(u_{i+1}^{-}\right)=-\left(a_{i+1} P_{i-2}+b_{i+1,0} P_{i-1}+c_{i+1,0} P_{i}\right)\left(2+\alpha_{i+1}\right)+\left(b_{i+1,1} P_{i-1}+c_{i+1,1} P_{i}\right)\left(2+\alpha_{i+1}\right) ; \\
& Q_{i}^{\prime \prime}\left(u_{i+1}^{+}\right)=2\left(b_{i 1} P_{i-2}+c_{i 1} P_{i-1}\right)+\left(b_{i 2} P_{i-2}+c_{i 2} P_{i-1}\right)\left[-6-\left(4 \beta_{i}+2\right)\right]+\left(b_{i 3} P_{i-2}+c_{i 3} P_{i-1}+d_{i} P_{i}\right)\left(4 \beta_{i}+2\right) ; \\
& Q_{i+1}^{\prime \prime}\left(u_{i+1}^{-}\right)=\left(a_{i+1} P_{i-2}+b_{i+1,0} P_{i-1}+c_{i+1,0} P_{i}\right)\left(4 \alpha_{i+1}+2\right)+\left(b_{i+1,1} P_{i-1}+c_{i+1,1} P_{i}\right)\left[-6-\left(4 \alpha_{i+1}+2\right)\right]+2\left(b_{i+1,2} P_{i-1}+c_{i+1,2} P_{i}\right) .
\end{aligned}
$$

According to the coefficient $a_{i}, b_{i, j}, c_{i, j}, d_{i}$ of expression (2) and lemma 1 , we can get $Q_{i}\left(u_{i+1}^{+}\right)=Q_{i+1}\left(u_{i+1}^{-}\right) ; Q_{i}^{\prime}\left(u_{i+1}^{+}\right)=Q_{i+1}^{\prime}\left(u_{i+1}^{-}\right) ; Q_{i}^{\prime \prime}\left(u_{i+1}^{+}\right)=Q_{i+1}^{\prime \prime}\left(u_{i+1}^{-}\right)$.

The theorem can be proved.

\subsection{Uniform CR-B spline image}

Figure 2 shows the uniform CR-B spline curve. (a) All graphs of $\alpha_{i}(i=3,4, \ldots, n)$ and $\beta_{i}(i=3,4, \ldots, n)$ are equal. (b) The black solid line in the figure shows the case that all 
Cubic Rational B-Spline Curves with Two Parameters

parameters are $\alpha_{i}=\beta_{i}=0$, the green dotted line shows that one of the parameters is adjusted to $\alpha_{i}=1$, and the blue dotted line shows that one of the parameters is adjusted to $\beta_{i}=1$.

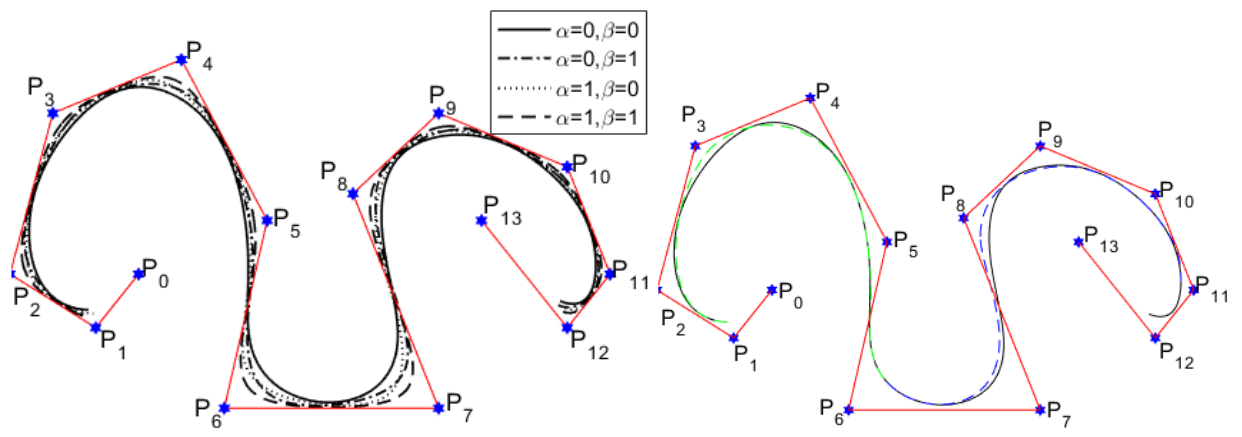

(a)

(b)

Figure 2: CR-B spline curve

\section{Conclusion}

In view of the problems existing in the improvement of the B-spline method in the traditional literature, such as the parameter does not have the global nature, the method does not have the generality, and can not retain the important properties of the traditional $\mathrm{B}$-spline method, this paper proposes a non-uniform rational B-spline base with two parameters to construct the curve. The example shows that the B-spline curve constructed in this paper solves the problems left over by the traditional improved literature, and is suitable In line with the curve design. In order to design a more practical shape, it is necessary to discuss the shape of the curve in detail, such as cusp, inflection point, heavy node, convexity, etc.

Acknowledgement. Research fund supported by National Natural Science Foundation of China (No. 61861040); Science and technology achievements transformation project of Gansu Provincial Department of Education (No.2017D-09).

The authors are thankful to the reviewers for their suggestions to improve the writing of the paper.

\section{REFERENCES}

1. G.C.Zhang and Z.X.Geng, $\alpha$ extension of the cubic uniform B-spline curve, Journal of Computer-Aided Design \& Computer Graphics, 7 (2007) 884-887.

2. X.L.Han and S.J.Liu, An extension of cubic uniform B-spline curve, Journal of Computer-Aided Design \& Computer Graphics, 5 (2003) 576-578.

3. W.T.Wang and G.Z.Wang, Uniform B-spline with shape parameter, Journal of Computer-Aided Design \& Computer Graphics, 6 (2004) 783-788.

4. X.Q.Wu and X.L.Han, Quadratic trigonometric polynomial Bézier curve with a shape parameter, Journal of Engineering Graphics, 1 (2008) 82-87.

5. L.H.Shi and G.C.Zhang, New extension of cubic TC-Bézier curves, Computer Engineering and Applications, 47(4) (2011) 201-204.

6. Y.G.Lyu, G.Z.Wang and X.N.Yang, Uniform hyperbolic polynomial B-spline curves, Computer Aided Geometric Design, 19(6) (2002) 379-393. 
Ji Peipei, Zhang Guicang and Wang Kai

7. X.M.Liu, W.X.Xu and Y.Guan, Hyperbolic polynomial uniform B-spline curves and surfaces with shape parameter, Graphical Models, 72(1) (2010) 1-6.

8. H.Y.Wu and H.Zuo, Quadratic non-uniform hyperbolic B-spline curves with multiple shape parameters, Journal of Computer-Aided Design \& Computer Graphics, 19(7) (2007) 876-883.

9. J.C.Li, L.Z.Song and C.Z.Liu, Cubic trigonometric pseudo B-spline combining multiple character-istics, Journal of Image and Graphics, 21(4) (2016) 425-433.

10. L.L.Yan and X.L.Han, High-order continuous shape adjustable trigonometric polynomial curve and surface, Journal of Image and Graphics, 20(03) (2015) 427-436.

11. Y.Jing, W.Fan and Tan Jieqing, A $C^{2}$ continuous unit quaternion Interpolatory spline curve, Journal of Image and Graphics, 29(01) (2017) 45-51.

12. L.L.Yan, X.L.Han and Z.Y.Rao, The $\lambda$-B curve design with a local shape parameter, Journal of Image and Graphics, 21(2) (2016) 174-183.

13. J.C.Li, L.L.Yan and C.Z.Liu, Quintic composite spline with adjustable shape and parameter selection, Journal of Image and Graphics, 22(2) (2017) 179-204.

14. L.L.Yan and X.L.Han, Conformal extension of cubic uniform B-spline curve, Application Research of Computers, 34(1) (2017) 295-301.

15. F.L.Chen, X.Q.Wu and X.Y.Zhu, Generalized cubic DP curve, Computer Science, 39(12) (2012) 264-267.

16. Z.K.Liu, Advanced Algebra, Beijing: Higher Publishing House Education, 2003.

17. G.Frain, Curves and Surfaces for Computer Aided Geometric Design, Academic Press, San Diego, 1993. 